\title{
Work in Progress - Undergraduate Research in Power Electronics - The Super Barrier Rectifier Diode Project
}

\author{
TaufikTaufik \\ Electrical Engineering Department, California Polytechnic State University \\ San Luis Obispo, CA 93407 taufik@calpoly.edu
}

\begin{abstract}
This paper presents an undergraduate student research project which involved a new power diode called the Super Barrier Rectifier (SBR) diode. Several projects incorporating SBR diodes in dc-dc converters performed by undergraduate students will be described. Challenges in conducting these projects along with results will also be discussed.
\end{abstract}

Index Terms - Undergraduate research.

\section{INTRODUCTION}

Power Electronics has recently experienced rapid growth due mainly to advances in semiconductor technology [1] as evidenced by the expansion of power semiconductor markets into areas such as automotive electronics, renewable energy, and portable electronics [2]. As a result, there has been an increasing demand for power electronic engineers. At $\mathrm{Cal}$ Poly, the demand has been noticeable by the rising number of companies that participated in campus career fair looking to hire graduating seniors with power electronic background. In turn, enrollment in power electronic courses at Cal Poly has increased in the past few years [3] and consequently so has the number of senior design projects in power electronics.

To better accommodate students wanting to conduct senior projects in power electronics, real-world design ideas were sought from local power semiconductor companies. One such company was APD Semiconductor who offered an undergraduate research involving a new type of power diode called the Super Barrier Rectifier (SBR) diode. The research was aimed to investigate the benefits of SBR in commonly used dc-dc converters. To meet this objective, 9 senior projects were conducted involving 15 undergraduate students.

\section{The SBR Research Project}

The two families of diodes widely used for power electronic applications are the pn-junction and Schottky diodes. Unfortunately, both diodes still possess physical limitations that preclude their use in certain applications. For example, pn-junction diodes are reliable at high voltage applications, but they are relatively slow. Hence, pn-junction diodes are not suitable for fast switching applications. On the other hand, Schottky diodes are fast but they are not available at high voltages. Table 1 lists typical trade-off comparison between the two diodes [4]:

TABLE 1

Performance comparison of pn and schottky

\begin{tabular}{|l|c|c|}
\hline & Pn-junction & Schottky \\
\hline Forward Voltage & High & Low \\
\hline Reverse Leakage Current & Low & High \\
\hline Breakdown Voltage Range & High & Medium \\
\hline Surge Reliability & High & Low \\
\hline Thermal Immunity & High & Low \\
\hline Switching speed & Low & High \\
\hline
\end{tabular}

As shown in Table 1, important operating characteristics are complimentary between the pn-junction diode and the Schottky diode. SBR diodes attempt to bridge this gap by uniquely capturing the benefits provided by each of these traditional devices and integrate them into a single component [5]. Other benefits of SBR which became the focus of the research project are their very low on-state losses and low leakage currents.

The SBR research project presented in this paper entailed the implementation and performance study of SBR diodes as compared with Schottky diodes in commonly used dc-dc converters. Several important circuit parameters that were investigated include overall converter's efficiency, power loss analysis due to forward conduction drop and leakage, and switching losses. Impact of thermal impedance to circuit's performance and effect of changing switching frequency to converter's efficiency were also studied. The following lists $9 \mathrm{dc}-\mathrm{dc}$ converters that were planned for the SBR research:

- Flyback Converter $3.3 \mathrm{~V}$ at $50 \mathrm{~W}$

- Synchronous Flyback Converter $3.3 \mathrm{~V}$ at $50 \mathrm{~W}$

- Synchronous Flyback Converter $15 \mathrm{~V}$ at $50 \mathrm{~W}$

- Buck Converter 3.3 or $5 \mathrm{~V}$ at $1 \mathrm{~W}$

- Buck Converter 3.3 V at $30 \mathrm{~W}$

- Synchronous Buck Converter 3.3 V at $30 \mathrm{~W}$

- Buck Converter $12 \mathrm{~V}$ at $30 \mathrm{~W}$

- Boost Converter $12 \mathrm{~V}$ at $15 \mathrm{~W}$

- Off-line Flyback Power Supply $15 \mathrm{~V}$ at $100 \mathrm{~W}$ 
The research project spanned for two quarters and each project was formally administered under senior project design course. Therefore, as part of the course requirements, students involved in the research project had to submit weekly status progress. By the end of the second quarter, besides submitting their final reports, the students had to demo their hardware projects to faculty advisor and representatives from APD semiconductor.

\section{RESULTS}

9 dc-dc converters were designed, built and tested for the SBR undergraduate research. Most of the converters built were fully operational while some were partly functioning. Figure 1 shows one converter that was successfully built for this research project. Figure 2 illustrates an example of the result obtained from circuit shown in Figure 1 in which SBR improved converter's overall efficiency of the converter. Overall, based on the results obtained and with the exception in a few cases, it can be concluded that SBR diodes did provide potential benefits over Schottky diodes. Hence, SBR diodes offer great potential in future power electronic applications.

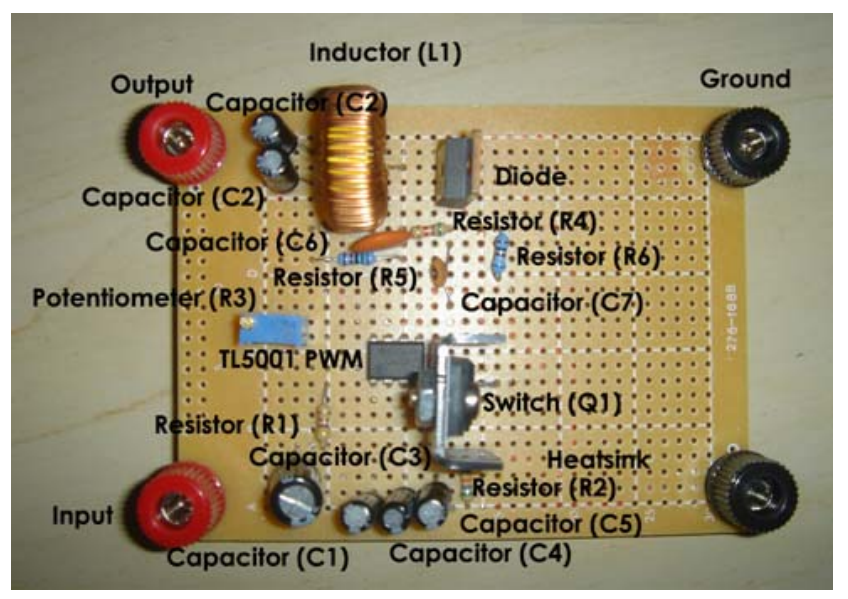

FIGURE 1

A $30 \mathrm{~W}$ Buck Converter

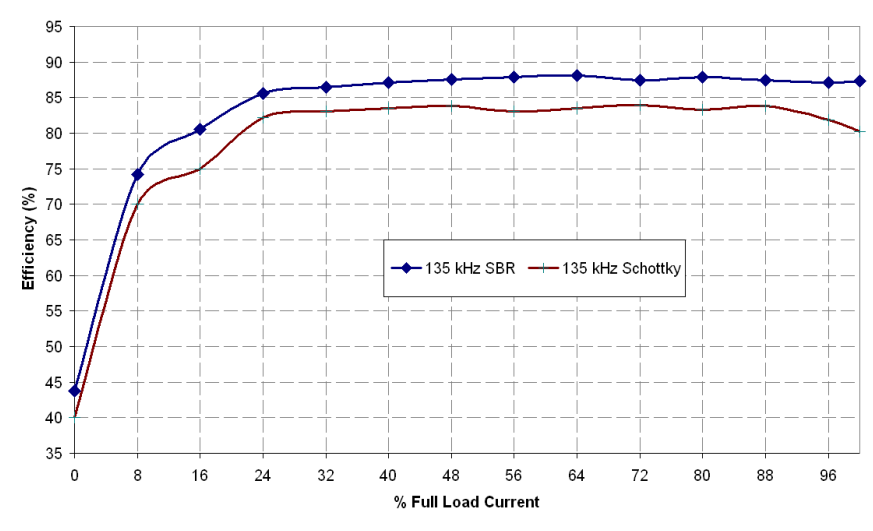

FIGURE 2

Overall efficiency using SBR vs. Schottky

\section{OBSTACleS AND FUTURE WORK}

There were several obstacles encountered during the course of the research activities. The first was equipment limitation. Although most tasks in this research could be performed using equipment currently available in the power electronics lab at Cal Poly; however, some measurements unfortunately could not be undertaken. An example was the lack of temperature chamber causing the inability to conduct below room temperature testing and measurements. The lack of sophisticated temperature measuring equipment also posed difficulty in conducting stable and accurate temperature measurements. Coordinating all 9 projects was also a challenge since 15 students were involved. It was very difficult to find a common time where all students involved could meet and report their progresses. To overcome this problem, it was decided that the students had to submit end of week status report via email. Another issue was the cost associated with building the $\mathrm{dc}-\mathrm{dc}$ converters since APD Semiconductor only supported the diodes needed for the research project.

Following the final demonstrations of the 9 projects before the representatives and engineers from APD Semiconductor, discussion on conducting more projects to further investigate SBR's performance in other power electronic applications took place.

\section{CONCLUSIONS}

This paper describes the undergraduate research performed at Cal Poly to investigate the performance of newly developed Super Barrier Rectifier diode. Students designed and built dcdc converters to test the performance of SBR diodes as compared to Schottky diodes. Overall, this research project was a success. More importantly, the research project provided the hands-on experience valuable for the students to better prepare them for the rapidly growing power semiconductor industry. Future collaboration with APD Semiconductor to further investigate SBR performance in many other power electronic applications was planned.

\section{REFERENCES}

[1]. P. Thollot, "Power Electronics Today", Proceedings of the 1990 IEEE Colloquium in South America, 1990, Pages 184-187.

[2]. Moguilnaia, N.A.; Vershinin, K.V.; Sweet, M.R.; Spulber, O.I.; De Souza, M.M.; Narayanan, E.M.S, "Innovation in Power Semiconductor Industry: Past and Future", IEEE Transactions on Engineering Management, volume 52, Issue 4, Nov. 2005 Page(s): $429-439$.

[3]. Taufik, "Power Electronics Courses That Work", Proceedings of 2006 Frontier in Education Conference, San Diego, Oct 2006.

[4]. "Super Barrier Rectifier: An Evolution In Power Rectifier Technology", Diodes Inc. website.

[5]. V. Rodov, A. Ankoudinov, Taufik, "Super Barrier Rectifier - A New Generation of Power Diode", Proceedings of 2007 American Power Electronics Conference, February 2007. 